\title{
Enhancing slow and fast light effects in quantum dot optical amplifiers through ultrafast dynamics
}

Chen, Yaohui; Mørk, Jesper

Published in:

Conference Proceedings, LEOS

Link to article, DOI:

10.1109/LEOS.2009.5343397

Publication date:

2009

Document Version

Publisher's PDF, also known as Version of record

Link back to DTU Orbit

Citation (APA):

Chen, Y., \& Mørk, J. (2009). Enhancing slow and fast light effects in quantum dot optical amplifiers through ultrafast dynamics. In Conference Proceedings, LEOS (pp. 662-663). IEEE.

https://doi.org/10.1109/LEOS.2009.5343397

\section{General rights}

Copyright and moral rights for the publications made accessible in the public portal are retained by the authors and/or other copyright owners and it is a condition of accessing publications that users recognise and abide by the legal requirements associated with these rights.

- Users may download and print one copy of any publication from the public portal for the purpose of private study or research.

- You may not further distribute the material or use it for any profit-making activity or commercial gain

- You may freely distribute the URL identifying the publication in the public portal 


\title{
Enhancing Slow and Fast Light Effects in Quantum Dot Optical Amplifiers through Ultrafast Dynamics
}

\author{
Yaohui Chen and Jesper Mørk \\ DTU Fotonik Department of Photonics Engineering, Technical University of Denmark, DK-2800, Kgs. Lyngby, Denmark
}

\begin{abstract}
We numerically demonstrate an important role of ultrafast carrier dynamics in quantum dot amplifiers in achieving tunable microwave phase shifts at frequencies beyond the limits of the carrier lifetime.
\end{abstract}

Quantum dot, Semiconductor optical amplifier, Slow light, Coherent polulation oscillation

\section{INTRODUCTION}

Controllable slow and fast light has been experimentally demonstrated in different active semiconductor waveguide devices at room temperature by employing the effect of coherent population oscillations (CPO) [1-3]. This effect can be generally described as a wave mixing phenomenon, where light are mediated by the complex susceptibility, which in semiconductor structures has contributions from various carrier dynamical processes. One of the remaining challenges for the applications of microwave photonics is to alleviate the fundamental limits of both phase shifts and modulation frequency [4-6], i.e., a bandwidth of several gigahertz is determined by the carrier lifetime. Recent calculations and experiments indicate that Quantum dot (QD) semiconductor optical amplifiers (SOAs) are especially attractive candidates because of the unique bias-current-dependent saturation mechanisms with large bandwidth (exceeding $40 \mathrm{GHz}$ ) due to the fast picosecond carrier dynamics between discrete QD bound states [7-11]. On the other hand, only modest phase shifts, peaking around a modulation frequency of $500 \mathrm{MHz}$ was measured for a QD SOAs [3]. This was ascribed to the subnanosecond carrier lifetime, which is also dominant in quantum well (QW) or bulk SOAs.

In this work, we numerically demonstrate that tunable phase shifting based on CPO effects can be achieved in QD SOAs at higher frequencies beyond the limits of carrier lifetime. The main new result compared to previous works is to address the potential contributions of ultrafast carrier dynamics to CPO mechanisms.

\section{PRINCIPLE AND MODEL}

Fig.1(a) shows a schematic configuration of a phase shifter based on CPO effects for sinusoidal intensity modulated (IM) envelopes of an optical carrier [1-6] in QD SOAs. By choosing the optical wavelength of the optical carrier with proper polarization control, the phase shifting due to CPO effects can be systematically investigated with minimized polarization effect. The degree of phase shifting can be tuned either by controlling the bias of the QD SOA or by varying the input optical power after the Erbium-doped fiber amplifier (EDFA). (a)

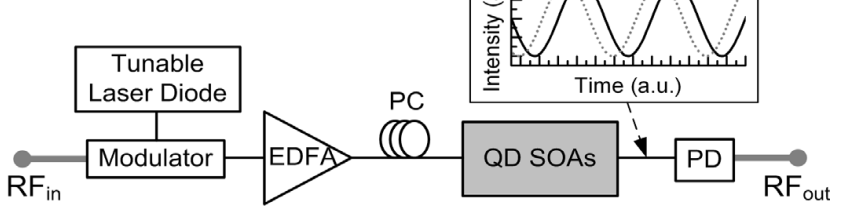

(b)

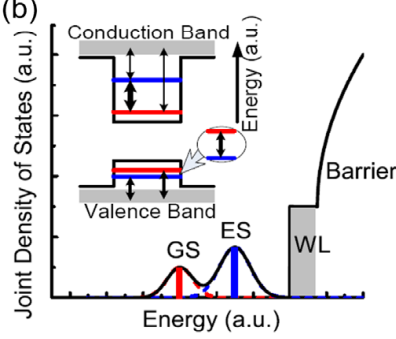

(c)

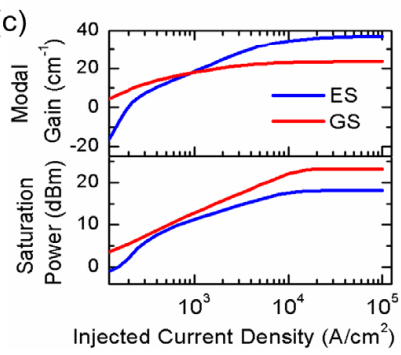

Fig. 1. Schematic diagram for the QD SOA based microwave phase shifter. (a) Configuration based on CPO effects in QD SOAs. PC: polarization controller; PD: photodetector. (b) Schematic diagram of inhomogeneously broadened QDs. (c) A simulated example of $\mathrm{CW}$ small signal gain and saturation power in QDs vs. injected current density.

The QD SOAs model is based on the implementation of a 3 -level rate equation model for carrier dynamics in $1100 \mathrm{~nm}$ InAs/GaAs QDs [8]. Fig. 1(b) shows a schematic diagram of inhomogeneously broadened QDs. Such model assumes that electron/holes from a common reservoir, wetting layer (WL) and barrier, can only occupy the two lowest discrete QD bound states, ground states (GS) and excited states (ES). Phenomenological rates for these capture/relaxation processes are defined to depend on the carrier density due to the phonon and Auger assisted contributions. The GS and ES carrier lifetime is fixed to $1 \mathrm{~ns}$, while the carrier lifetime in the reservoir has non-radiative contributions. For electron dynamics in the small signal regime at strong current injection levels $\left(10 \mathrm{kA} / \mathrm{cm}^{2}\right)$, the longest time is the reservoir carrier lifetime $(0.5 \mathrm{~ns})$, the intermediate time is the electron capture from WL to ES or GS (2.5ps), and the shortest time is the intradot electron relaxation time from ES to GS $(0.2 \mathrm{ps})$. The values are extracted from two-color pump-probe measurements [12]. A local carrier density description of QD bounded hole states with $100 \mathrm{fs}$ valence intra-band scattering time and a common valence band quasi-equilibrium level has been used to evaluate the contribution of hole dynamics to the optical gain. Fig. 1(c) shows the simulated small $\mathrm{CW}$ modal gain and saturation power in the QDs for the optical transition at the centre of GS and ES transition for different values of the injected current density. In order to compare the phase shifting results at 
different optical transitions, we use a reference current density $\left(1 \mathrm{kA} / \mathrm{cm}^{2}\right)$, at which modal gains for the compared optical transitions are similar. The device is $2 \mathrm{~mm}$ long and has $6 \mathrm{~cm}^{-1}$ internal loss.

\section{MODELLING RESULTS}
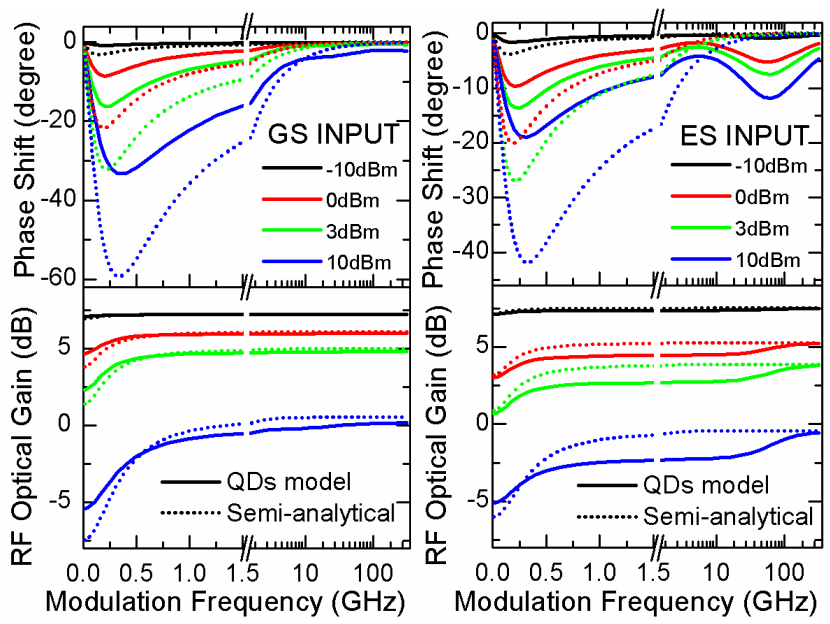

Fig. 2. Phase shift and RF optical gain as a function of modulation frequency at optical transitions corresponding to GS (left) and ES (right) for different input pump powers. Solid curves are based on the complete QD SOA model. Dotted curves are based on semi-analytical models used in $[3,6]$. The injected current density is $1 \mathrm{kA} / \mathrm{cm}^{2}$.

Fig. 2 shows the simulated phase shift and RF optical gain as a function of modulation frequency at different optical transitions for different input pump powers. Fig. 2 (left) is for the GS transition. A maximum phase shift of around $-34^{\circ}$ is observed at a peak modulation frequency around $300 \mathrm{MHz}$, which corresponds to a steep RF optical gain variation. As modulation frequency increases, phase shifts declines, which matches the experimental observation [3] and has been well explained by the carrier lifetime dominated by carrier density depletion (CDP) with a semi-analytical model [2,3,6]. Dotted curves as reference are semi-analytical results calculated with the constant carrier lifetimes and CW saturation power directly from QDs model. The quantity mismatch of phase shifting and gain in low frequency regime is due to the overestimation under the linear gain assumption in semi-analytical model. As we shifted to the case for ES transition as shown in Fig. 2 (right), we achieved phase shifting peaked at two distinctively separated frequencies, a value of around $-10^{\circ}$ peaked around $70 \mathrm{GHz}$ as well as a value of around $-18^{\circ}$ peak around $300 \mathrm{MHz}$. Meanwhile these two phase shift peaks corresponding to the two different plateau-levels of RF optical gain seen in the lower plot of Fig. 2. The second peak appearing at a much higher modulation frequency corresponds to the inverse of the carrier capture time of several picoseconds. This effect is not taken into account in the semi-analytical model due to the exclusion of ultrafast dynamics [6]. As a comparison, the intraband scattering dominated spectral hole burning (SHB) dynamics in bulk or QW is at the time scale around 50fs. In QDs, the interband scattering dominated carrier capture process is at least two or three orders faster than CDP process and one or two orders slower than the SHB process in bulk or QW. The ultrafast carrier dynamics in QD SOAs might strongly depend on the bias control condition and signal power as well as fabrication process, via the intersubband scattering times, which provides potential for controlling the second peak modulation frequency. Because of the different saturation powers for GS and ES transitions, even with similar modal gain, the quantity of GS phase shift is different from the case of ES. The maximum value of the phase shift can be further optimized by different control schemes and effects, such as influence from internal loss.

\section{CONCLUSION}

As the first proof of concept, we have numerically demonstrated that tunable phase shifting based on slow and fast light effects can be achieved in QD SOAs at modulation frequencies much higher than considered so far. This result is of importance for the implementation of QD SOAs based phase shifters at microwave frequencies like the $\mathrm{V}$ or $\mathrm{W}$ band.

\section{ACKNOWLEGEMENT}

The authors acknowledge support from the GOSPEL project financed by the European Commission, the QUEST project financed by the Danish Research Councils and the NATEC centre funded by VILLUM KANN RASMUSSEN FONDEN.

\section{REFERENCES}

[1] C. Chang-Hasnain amd S.L. Chuang, "Slow and fast light in semiconductor quantum-well and quantum-dot devices", J. Lightw. Technol., 24, 4642-4654, 2006

[2] J. Mørk et al., "Slow and fast light: Controlling the speed of light using semiconductor waveguides", Laser \& Photonics Reviews, 2008, Wiley VCH Verlag GmbH \& Co. kGaA, pp. 1-15

[3] A. Matsudaira et al., "Electrically tunable slow and fast lights in a quantum-dot semiconductor optical amplifier near $1.55 \mu \mathrm{m}$ ", Opt. Lett., 32, 2894-2896, 2007

[4] F. Öhman, K. Yvind, and J. Mørk, "Slow light in a semiconductor waveguide for true-time delay applications in microwave photonics", Photon. Technol. Lett., 19, 1145-1147, 2007

[5] W. Xue, Y. Chen, F. Öhman, S. Sales, and J. Mørk, "Enhancing light slow-down in semiconductor optical amplifiers by optical filtering", Opt. Lett., 21, 1084-1086, 2008

[6] Y. Chen, W. Xue, F. Öhman, and J. Mørk, "Theory of optical-filltering enhanced slow and fast light effects in semicondcutor optical waveguides", J. Lightw. Technol., 26, 3734-3743, 2008

[7] M. Sugawara, H. Ebe, N. Hatori and M. Ishida, "Theory of optical signal amplification and processing by quantum-dot semiconductor optical amplifiers", Phys. Rev. B, 69, 235332, 2004

[8] T. Berg and J. Mørk, "Saturation and noise properties in semiconductor optical amplifers", J. Quantum. Electron., 40, 1527-1539,2004

[9] A.V. Uskov, E.P. O'Reilly, M. Laemmlin, N.N. Ledntsov, D. Bimberg, "On gain saturation in quantum dot semiconductor optical amplifiers", Opt. Comm., 248, 211-219, 2005

[10] T. Akiyama, M. Sugawara, and Y. Arakawa, "Quantum-dot semiconductor optical amplifiers", Proceeding of IEEE, 95, 1757-1766, 2007

[11] C. Meuer et al., "High-speed small-signal cross-gain modulation in quantum-dot semiconductor optical amplifiers at $1.3 \mu \mathrm{m},, J$. Sel. Topics Quantum Electron., 15, 749-756, 2009

[12] I. O'Driscoll et al., "Electron and hole dynamics of InAs/GaAs quantum dot semiconductor optical amplifier", Appl., Phys. Lett., 91, 071111, 2007 